\title{
Early social environment affects the endogenous oxytocin system: a review and future directions
}

\author{
Emily Alves ${ }^{1}{ }^{*}$, Andrea Fielder ${ }^{2}$, Nerelle Ghabriel ${ }^{2,3}$, Michael Sawyer $^{4,5}$ and Femke T.A. Buisman-Pijlman ${ }^{1 *}$ \\ 1 School of Medical Sciences, University of Adelaide, Adelaide, SA, Australia \\ 2 School of Midwifery, University of South Australia, Adelaide, SA, Australia \\ ${ }^{3}$ School of Psychology, University of South Australia, Adelaide, SA, Australia \\ ${ }^{4}$ School of Paediatrics and Reproductive Health, University of Adelaide, Adelaide, SA, Australia \\ ${ }^{5}$ Research and Evaluation Unit, Women's and Children's Health Network, Adelaide, SA, Australia
}

\section{Edited by:}

Hubert Vaudry, University of Rouen,

France

Reviewed by:

Charles W. Wilkinson, University of

Washington, USA

Thiago Pereira Henriques,

Universidade Federal do Rio Grande do Sul, Brazil

\section{*Correspondence}

Emily Alves and Femke T.A.

Buisman-Pijlman, Adelaide University,

30 Frome Road, Adelaide, SA 5000,

Australia

e-mail: emily.alves@student.adelaide.

edu.au;

femke.buisman-pijlman@adelaide. edu.au
Endogenous oxytocin plays an important role in a wide range of human functions including birth, milk ejection during lactation, and facilitation of social interaction. There is increasing evidence that both variations in the oxytocin receptor (OXTR) and concentrations of oxytocin are associated with differences in these functions. The causes for the differences that have been observed in tonic and stimulated oxytocin release remain unclear. Previous reviews have suggested that across the life course, these differences may be due to individual factors, e.g., genetic variation (of the OXTR), age or sex, or be the result of early environmental influences, such as social experiences, stress, or trauma partly by inducing epigenetic changes. This review has three aims. First, we briefly discuss the endogenous oxytocin system, including physiology, development, individual differences, and function. Second, current models describing the relationship between the early life environment and the development of the oxytocin system in humans and animals are discussed. Finally, we describe research designs that can be used to investigate the effects of the early environment on the oxytocin system, identifying specific areas of research that need further attention.

Keywords: oxytocin, early-life environment, research design, individual differences, mother-infant bonding

\section{THE ENDOGENOUS OXYTOCIN SYSTEM}

Oxytocin, a mammalian hormone, is a naturally produced neuropeptide with nine amino acids. Oxytocin is mainly produced in magnocellular neurons in the hypothamalic paraventricular and supraoptic nuclei (1). The magnocellular neurons release oxytocin into circulating blood via the pituitary gland, while the parvocellular oxytocin neurons release oxytocin in other areas of the central nervous system (CNS). Recent evidence has demonstrated that oxytocinergic axons ending in forebrain regions including the central amygdala and nucleus accumbens may originate exclusively in the magnocellular neurons of the paraventricular nucleus (2). Evidence also indicates that central projections of hypothalamic oxytocin neurons may be widespread, and that oxytocin release from local axonal endings may be able to control regionassociated behaviors (2). Oxytocin is also produced in a number of peripheral tissues and organs, such as the uterus, ovaries, testis, vascular endothelium, and the heart (3). For a review of the anatomy and functional aspects of the oxytocin system, see Kiss and Mikkelson (4).

Oxytocin is released in response to a range of internal and external stimuli. Historically, it is known that oxytocin is released in response to vaginocervical and nipple stimulation and plays important roles in mammalian uterine contraction and lactation. Oxytocin also plays a crucial role in the milk ejection reflex. In lactating women, oxytocin peaks during the morning and then declines until the beginning of the afternoon, and it is released in a cyclic manner, with salivary oxytocin concentrations at their highest within 30 min before feeding begins (5). This indicates that the brain may be able to release oxytocin in anticipation of future behaviors and interactions.

More recent data demonstrate the pro-social role of oxytocin, including its role in social and emotional regulation (6), orgasm (7), regulating stress, and anxiety and facilitation of pair, maternal and infant bonding (8-12). Interestingly, the release of oxytocin is dependent on the social context. For example, calves more readily release oxytocin when suckling milk directly from their mother's udder than when drinking the same milk from a bucket (13). Another example shows that oxytocin administration combined with psychological support of a friend lowered salivary cortisol concentrations and was correlated with decreased anxiety and increased calmness in human males (8).

Large individual differences exist in basal concentrations of oxytocin and in response to stimulation [for a review, see Ref. (12)]. Basal individual oxytocin concentrations in humans have been found to vary by thousands of $\mathrm{pg} / \mathrm{ml}$ within the same study (14). Individual differences, such as genetic variation of the oxytocin receptor (OXTR) may explain some of the variation. Expression of the OXTR also varies in a sex-specific way. It is therefore possible that an interaction with the estrogen system plays a role that warrants further attention and investigation (15).

\section{OXYTOCIN AND THE EARLY SOCIAL ENVIRONMENT}

The early-life environment is critical for development in humans and other mammals [for a review on the long-term impact of 
early-life events, see Ref. (16)]. A significant component of the early-life environment is mother-infant bonding. Social experiences in the early-life period and bonding form the basis for healthy social and emotional development, possibly of the mechanism that manages stress and resilience (11). The well-documented protective associations between secure attachments and later social functioning and behavior underscore the need to understand the origins of attachments and identify the specific individual differences that influence attachment and development (17). Current research highlights the link between oxytocin concentrations and a specific set of maternal bonding behaviors and attitudes in humans (18-24). There is evidence to suggest that oxytocin plays an important role in facilitation of mother-infant bonding [for reviews, see Ref. $(10,25)]$ and that the early social environment can shape the developing oxytocin system. There is substantial literature documenting the relationship between oxytocin and the early-life environment in animals (26-28); however, minimal research has focused on this area in humans. Importantly, there is only limited data about the normal development of the oxytocin system in humans over time (11).

Oxytocin plays an important role in priming mammals to form social bonds, but in turn, the early social environment may also be able to shape the development of the oxytocin system [for a review, see Ref. (11)]. Interestingly, studies have demonstrated correlations between infant and parental oxytocin concentrations and parenting behaviors in child-parent interactions $(14,29)$. The following section will review the influence of prenatal and postnatal environment on the oxytocin system. Additionally, maternal mental health problems will be addressed as they can negatively affect the mother-infant bond.

\section{PRENATAL ENVIRONMENT}

In humans, prenatal stressors (including substance use, maternal depression, and chronic stress) can lead to a range of abnormal neurodevelopmental outcomes for infants [for a review, see Ref. (30)]. A recent human study found that male and female fetuses respond differentially to chronic maternal stress, indicating that sex may be an important factor influencing fetal development (31).

Lee and colleagues (32) demonstrated that a regime of prenatal stressors in pregnant rats caused numerous changes in the oxytocin systems of adult male offspring (less oxytocin mRNA in the paraventricular nucleus, but increased OXTR binding in the central amygdala). Prenatally stressed rats showed disturbed social behavior, which could be normalized with local oxytocin administration in the central amygdala. Cross fostering the pups did not normalize the behavior.

Drug abuse, including smoking, heavy consumption of alcohol, and illegal substances, can adversely affect fetal development and can also impair infant health and development. Alcohol can cause numerous growth impairments, and is especially well known for its harmful effects on the developing nervous system. Tobacco and cannabis smoking are also notorious for fetal growth-restricting and gestation-shortening effects (33). There is ample evidence on the long-term effects of prenatal drug use (ethanol, stimulants, and nicotine) on rodent offspring, focusing on social behavior and drug use and changes to the oxytocin system (33). Exposure to cocaine in the prenatal period in rats affects both the oxytocin system and social behavior and increases susceptibility to addiction later in life $(34,35)$.

\section{POSTNATAL ENVIRONMENT}

The oxytocin system continues to develop after birth, and this development may be critical for providing humans with the skills for healthy social functioning (25). There is extensive research focusing on the long-term effects of early-life adversity and social environment on the oxytocin system; however, most of these studies use animal models. Differences in rearing conditions and bonding behavior can influence adult social and parental behavior in prairie voles (9). Additionally, quality of maternal behavior has been linked to differences in oxytocin concentrations and OXTR expression observed in animal models $(9,36)$. For example, high levels of maternal licking resulted in increased plasma oxytocin concentrations in neonatal rats (37). Furthermore, Kojima and colleagues (38) found that maternal skin-to-skin contact stimulates rat pups' central oxytocin concentrations. Early-life adversity and differences in the early social environment may also adversely affect the expression and concentration of the OXTR. Veenema (26) provides a thorough review on the effects of early-life manipulations in rodents on the distribution and expression of oxytocin and vasopressin receptors. Bales and Perkeybile (39) also provide a good review of the effect of early experience on the OXTR system.

Human studies with infants and their parents have demonstrated how oxytocin levels rise in response to social interaction and how infant and parental oxytocin concentrations correlate (39). These findings have been supported in previous research measuring cerebrospinal fluid (CSF) oxytocin concentrations, which have shown that higher infant CSF oxytocin concentrations were positively correlated with active initiation and interest in parental social interaction (40). The human oxytocin system seems to be receptive to both positive and negative early social experiences, such as separation (41). Infants who experienced high affect parent-infant synchrony (i.e., monitoring and responding) showed increase oxytocin saliva measures compared to infants reared in the presence of low affect parent-infant synchrony (42). This suggests that the early environment may directly affect peripheral oxytocin concentrations in humans. Importantly, a study by Wismer Fries and colleagues (43) reported significant social deficiencies and low oxytocin concentrations in children reared in extremely aberrant social environments. These results indicate that the early environment may influence cross-generation transmission of human social attachments and behavior. They also support the notion that peripheral oxytocin measurements may be correlated to social behavior in both infants and adults.

\section{MATERNAL ANXIETY, STRESS, AND DEPRESSION}

Maternal mental health problems can greatly influence the mother-child bond as they affect the way they perceive their child's needs and cues, their stress resilience, and their general emotional availability.

Research has shown that mothers with postpartum anxiety report significantly lower bonding with their infants than healthy mothers (44). Depressed mothers are more likely to perceive their infant's behavior negatively than healthy mothers (45). Depression 
can also play an important role in influencing the early-life environment through its effect on maternal behavior and motherinfant bonding. Research shows that the most significant predictor of lower postnatal maternal attachment was depressive symptoms experienced during the final stages of pregnancy and in the postnatal period $(18,46)$. Despite this emerging evidence, a recent review of the literature (47) identifies that mechanisms underlying maternal stress, depression and anxiety, and their effects on infant outcomes are poorly understood. Due to its important role in mother-infant bonding, oxytocin could be involved in these mechanisms; however, further study is needed.

Interestingly, a recent pilot study found that exogenous administration of oxytocin stimulated protective behavior in mothers with postnatal depression (48). Eapen and colleagues (49) also found an association between lower plasma oxytocin levels in the post partum period and separation anxiety and depression during pregnancy. Further study is needed to determine the relationship between maternal depression and oxytocin, how this relationship may affect infants, and the potential role of exogenous oxytocin in treating depression.

\section{CONSIDERATIONS FOR RESEARCH}

The previous sections have introduced the endogenous oxytocin system and its relationship to early environmental and social factors. The present section will identify limitations in the current literature and provide suggestions for future research.

This review has established that there is evidence showing that early social environment in animals is correlated with altered oxytocin concentrations. Particular attention to maternal behaviors during the postnatal period is warranted given the established link between mother-infant dyadic interactions and later physical, emotional, and social health. Studies by Feldman and colleagues $(14,18,41,42)$ have provided insight into the range of saliva and plasma oxytocin concentrations that should be expected in both mothers and babies. However, very little is still known about the normal development of the oxytocin system into childhood and adolescence.

Additionally, a proposed direction of research would be to determine how an early adverse social environment in humans affects the oxytocin system. Of interest are changes in basal concentrations, differences in reactivity of the oxytocin system under stress or social interaction and changes in OXTR characteristics (e.g., investigation of epigenetics, binding affinity, up- or downregulation of receptors, and methylation).

Up- or downregulation of the number of OXTRs, localization and sensitivity of OXTRs are currently difficult to research in humans, as there is no radio-active ligand that can be used in this process. Therefore, a number of different research techniques are needed to investigate these changes through other means, including the collection of biological samples during observational studies and psychological testing undertaken in large cohort studies, and further refinements of assay methodologies.

Epigenetic changes will be of interest to investigate when determining positive or negative effects of early social environment on the developing oxytocin system. Epigenetics refers to the regulation of DNA transcription without alteration of the original sequence. DNA methylation is an important epigenetic modification in response to, e.g., oxygen deprivation, trauma, or drug use. A recent study found that traumatic experiences and stressful life events in early life were associated with higher methylation of the NR3C1 gene (50). Although the topic of epigenetics has not been thoroughly explored in the present review, there is promising research indicating its importance $(28,51-53)$.

\section{SAMPLE COLLECTION AND MEASUREMENT}

Extensive discussion in the field addresses the most suitable and reliable method of collecting and analyzing oxytocin samples, both from the periphery and centrally. Different methods of sample collection and measurement contribute to the large range in oxytocin concentrations reported in the literature (54).

Human research into oxytocin is significantly more difficult than animals due to the inability to non-invasively assess brain oxytocin pathways and concentration. However, peripheral oxytocin concentrations can be tested through plasma, urine or saliva. These measures possibly may not directly correspond with brain concentrations; however, research has shown that changes in behavior are linked to changes in peripheral oxytocin concentrations (14, 54). There is also increasing evidence suggesting that peripheral oxytocin is reflective of changes in central concentrations. For example, a recent study of human children found that plasma oxytocin concentrations significantly and positively predicted CSF oxytocin concentrations (55).

Collecting samples of oxytocin from infants provide an additional challenge to researchers. CSF, urine, and plasma samples are generally unsuitable in these situations as the collection method is invasive and painful; furthermore, it is unlikely that parents will provide consent for their infant's participation in a study with these extraction methods. Finding correlations between oxytocin concentrations in urine and social behavior has shown some success in both animal and human studies $(56,57)$. However, this collection method may still not be appropriate when attempting to determine an acute infant increase in oxytocin in response to a parent-infant interaction, as urine concentrations represent accumulated oxytocin concentrations and are not always readily available for collection, especially in infants. Saliva, therefore, would appear to be the most ideal method of measuring oxytocin concentrations in infants, with adoption of this method resulting in significant associations between infant and parent oxytocin concentrations and social behavior (42). Recent research from our group (unpublished) has supported previous studies, finding that oxytocin concentrations can be determined from both adult and infant saliva samples. Adults are able to provide this sample by simply spitting into a tube. Saliva from infants can be collected by collecting passive drool from the baby's mouth with a syringe and then dispensing into a tube. Parents have also willingly provided infant consent for this form of collection as it is a minimally invasive and completely painless extraction method. A limitation of this process, however, is that some infants are more accepting of syringing than others, influencing the collection time. This process of collection could also potentially be difficult if the infant is distressed.

Further refinement of oxytocin assay methodologies is also needed. A comparison of enzyme immunoassays (EIA) and radio immunoassays (RIA) of human plasma samples with and without 
extraction found that without extraction, plasma measured by EIA was more than 100 times higher than in extracted plasma, and the correlation between them was minimal $(p=0.54)(58)$. The same study found that when using an RIA, the majority of samples (90\%) were below the level of detection. This could provide some insight into the general inconsistency of results across different studies, indicating the need to refine oxytocin assay methodologies. Currently, comparing group differences within experiments is the most reliable approach.

Specificity of the role of oxytocin provides a challenge in determining the link with early adversity. Oxytocin and vasopressin are very similar in structure, and the two hormones have a high affinity for each other's receptors (59). It is suggested that early social experiences influence sensitivity to both the oxytocin and vasopressin systems (28). This emphasizes the need for further understanding of the systems interacting with oxytocin and the relationship they may have with early adversity and development in humans.

While focusing on the effect of early life social experiences on infants and their developing oxytocin system, it is important to acknowledge that the oxytocin system has numerous bilateral interactions (e.g., dopamine and HPA-axis) that are likely to affect behavior [for a review, see Ref. (12)]. Changes to the oxytocin system will also influence these systems and vice versa. Additionally, systems like the HPA-axis will be affected by environmental influences as well. Researchers focusing on long-term effects of early social environments need to keep these considerations in mind and try to address all the dimensions.

\section{CHARACTERIZATION AND MEASUREMENT OF EARLY ADVERSITY}

Another research challenge is measuring early adversity itself. First, there is little consensus on the term, and second, it is conceptualized differently across different disciplines, i.e., neglect, abuse, toxic stress, disorganized attachment, reduced bonding, etc. Detecting and quantifying early adversity is challenging when relying on questionnaires. Observing single mother-child interactions in a laboratory setting or home environment can provide great insight into the early social environment. Assessing attachment status is often not an option, as extensive training is needed. Mothers of infants who may be experiencing early adversity may be reluctant to participate in behavioral observations, although large cohort studies may be able to incorporate this in sub-groups. The next challenge is selecting a tool that can be used to score behavior in a dyad and quality of the interaction (e.g., reciprocity), which is objective, intuitive, and has large inter-rater reliability. There are few scales that have been validated for this purpose meeting all these criteria.

\section{CONCLUDING REMARIKS}

Review of the literature indicates that the early-life is a vital period for healthy development in humans, and that oxytocin is an important regulator of emotional development. Specifically, investigating the effect of early adversity on the endogenous oxytocin system is important in understanding normal behavior and social disorders. Understanding this effect can increase knowledge of how the early environment appears to change the way humans are hardwired to respond in social and stressful situations.
A number of animal studies have been conducted; however, more human research specifically focusing on this relationship is necessary incorporating a large range of study designs. Future studies must consider the obstacles that prevent conducting this research, such as collecting appropriate peripheral oxytocin measures and selecting a suitable tool to score both infant and maternal behavior.

\section{REFERENCES}

1. Lim MM, Young LJ. Neuropeptidergic regulation of affiliative behaviour and social bonding in animals. Horm Behav (2006) 50(4):506-17. doi:10.1016/j. yhbeh.2006.06.028

2. Knobloch HS, Charlet A, Hoffman LC, Eliava M, Khrulev S, Cetin AH, et al. Evoked axonal oxytocin release in the central amygdala attenuates fear response. Neuron (2012) 73(3):553-66. doi:10.1016/j.neuron.2011.11.030

3. Nishimori K, Takayanagi Y, Yoshida M, Kasahara Y, Young LJ, Kawamata M. New aspects of oxytocin receptor function revealed by knockout mice: sociosexual behaviour and control of energy balance. Prog Brain Res (2008) 170:79-90. doi:10.1016/S0079-6123(08)00408-1

4. Kiss A, Mikkelson JD. Oxytocin - anatomy and functional assignments: a minireview. Endocr Regul (2005) 39(3):97-105.

5. White-Traut R, Watanabe K, Pournajafi-Nazarloo H, Schwertz D, Bell A, Carter S. Detection of salivary oxytocin levels in lactating women. Dev Psychobiol (2009) 51(1):367-73. doi:10.1002/dev.20376

6. Neumann ID. Brain oxytocin: a key regulator of emotional and social behaviour in both females and males. J Neuroendocrinol (2008) 6:858-65. doi:10.1111/j. 1365-2826.2008.01726.x

7. Magon N, Kalra S. The orgasmic history of oxytocin: love, lust, and labor. Indian J Endocrinol Metab (2011) 15(Suppl 3):S156-61. doi:10.4103/2230-8210.84851

8. Heinrichs M, Baumgartner T, Kirschbaum C, Ehlert U. Social support and oxytocin interact to suppress cortisol and subjective responses to psychosocial stress. Biol Psychiatry (2003) 54(12):1389-98. doi:10.1016/S0006-3223(03)00465-7

9. Ahern TH, Young LJ. The impact of early life family structure on adult social attachment, alloparental behaviour, and the neuropeptide systems regulating affiliative behaviours in the monogamous prairie vole (microtus ochrogaster). Front Behav Neurosci (2009) 3:17. doi:10.3389/neuro.08.017.2009

10. Olff M, Frijling JL, Kubzansky LD, Bradley B, Ellenbogen MA, Cardoso C, et al. The role of oxytocin in social bonding, stress regulation and mental health: an update on the moderating effects of context and interindividual differences. Psychoneuroendocrinology (2013) 38(9):1883-94. doi:10.1016/j.psyneuen.2013. 06.019

11. Tops M, Koole SL, IJzerman H, Buisman-Pijlman FTA. Why social attachment and oxytocin protect against addiction and stress: insights from the dynamics between ventral and dorsal corticostriatal systems. Pharmacol Biochem Behav (2014) 119:39-48. doi:10.1016/j.pbb.2013.07.015

12. Buisman-Pijlman FT, Sumracki NM, Gordon JJ, Hull PR, Carter CS, Tops M. Individual differences underlying susceptibility to addiction: role for the endogenous oxytocin system. Pharmacol Biochem Behav (2014) 119(1):22-38. doi:10.1016/j.pbb.2013.09.005

13. Lupoli B, Johansson B, Uvnäs-Moberg K, Svennersten-Sjaunja K. Effect of suckling on the release of oxytocin, prolactin, cortisol, gastrin, cholecystokinin, somatostatin and insulin in dairy cows and their calves. J Dairy Res (2001) 68(2):175-87. doi:10.1017/S0022029901004721

14. Feldman R, Gordon I, Zagoory-Sharon O. Maternal and paternal plasma salivary and urinary oxytocin and parent-infant synchrony: considering stress and affiliation components of human bonding. Dev Sci (2011) 14(4):752-61. doi:10.1111/j.1467-7687.2010.01021.x

15. Murakami G, Hunter RG, Fontaine C, Ribeiro A, Pfaff D. Relationships among estrogen receptor, oxytocin and vasopressin gene expression and social interaction in male mice. Eur J Neurosci (2011) 34(3):469-77. doi:10.1111/j.14609568.2011.07761.x

16. Boersma GJ, Bale TL, Casanello P, Lara HE, Lucion AB, Suchecki D, et al. Longterm impact of early-life events on physiology and behaviour. J Neuroendocrinol (2014) 26(9):587-602. doi:10.1111/jne.12153

17. Wong MS, Mangelsdorf SC, Brown GL, Neff C, Schoppe-Sullivan SJ. Parental beliefs, infant temperament, and marital quality: associations with infantmother and infant-father attachment. J Fam Psychol (2009) 23(6):828-38. doi:10.1037/a0016491 
18. Feldman R, Weller A, Zagoory-Sharon O, Levine A. Evidence for a neuroendocrinological foundation of human affiliation: plasma oxytocin levels across pregnancy and the postpartum period predict mother-infant bonding. Psychol Sci (2007) 18(11):965-70. doi:10.1111/j.1467-9280.2007.02010.x

19. Strathearn L, Fonagy P, Amico J, Montague PR. Adult attachment predicts maternal brain and oxytocin response to infant cues. Neuropsychopharmacology (2009) 34(13):2655-6. doi:10.1038/npp.2009.103

20. Gordon I, Zagoory-Sharon O, Leckman JF, Feldman R. Oxytocin, cortisol, and triadic family interactions. Physiol Behav (2010) 101(5):679-84. doi:10.1016/j. physbeh.2010.08.008

21. Atzil S, Hendler T, Feldman R. Specifying the neurobiological basis of human attachment: brain, hormones, and behavior in synchronous and intrusive mothers. Neuropsychopharmacology (2011) 36(13):2603-15. doi:10.1038/npp. 2011.172

22. Atzil S, Hendler T, Zagoory-Sharon O, Winetraub Y, Feldman R. Synchrony and specificity in the maternal and the paternal brain: relations to oxytocin and vasopressin. J Am Acad Child Adolesc Psychiatry (2012) 51(8):798-811. doi:10.1016/j.jaac.2012.06.008

23. Feldman R, Gordon I, Influs M, Gutbir T, Ebstein RP. Parental oxytocin and early care giving jointly shape children's oxytocin response and social reciprocity. Neuropsychopharmacology (2013) 38(7):1154-62. doi:10.1038/npp.2013.22

24. Kim S, Fonagy P, Koos O, Dorsett K, Strathearn L. Maternal oxytocin response predicts mother-to-infant gaze. Brain Res (2014) 1580:133-42. doi:10.1016/j. brainres.2013.10.050

25. Taylor AE, Lee HE, Buisman-Pijlman FTA. Oxytocin treatment in paediatric populations. Front Behav Neurosci (2014) 8:360. doi:10.3389/fnbeh.2014.00360

26. Veenema AH. Toward understanding how early-life social experiences alter oxytocin- and vasopressin-regulated social behaviours. Horm Behav (2011) 61(3):304-12. doi:10.1016/j.yhbeh.2011.12.002

27. Bales KL, Boone E, Epperson P, Hoffman G, Carter C. Are behavioural effects of early experience mediated by oxytocin? Front Psychiatry (2011) 2:24. doi:10.3389/fpsyt.2011.00024

28. Cushing BS, Kramer KM. Mechanisms underlying epigenetic effects of early social experience: the role of neuropeptides and steroids. Neurosci Biobehav Rev (2005) 29(7):1089-105. doi:10.1016/j.neubiorev.2005.04.001

29. Levine A, Zagoory-Sharon O, Feldman R, Weller A. Oxytocin during pregnancy and early postpartum: individual patterns and maternal-foetal attachment. Peptides (2007) 28(6):1162-9. doi:10.1016/j.peptides.2007.04.016

30. O'Donnell K, O'Connor TG, Glover V. Prenatal stress and neurodevelopment of the child: focus on the HPA axis and role of the placenta. Dev Neurosci (2009) 31:285-92. doi:10.1159/000216539

31. Wainstock T, Shoham-Vardi I, Glasser S, Anteby E, Lerner-Geva L. Fetal sex modifies effects of prenatal stress exposure and adverse birth outcomes. Stress (2014):1-8. doi:10.3109/10253890.2014.974153

32. Lee PR, Brady DL, Shapiro RA, Dorsa DM, Koenig JI. Prenatal stress generates deficits in rat social behaviour: reversal by oxytocin. Brain Res (2007) 2(1156):152-67. doi:10.1016/j.brainres.2007.04.042

33. Vaiserman AM. Long-term health consequences of early-life exposure to substance abuse: an epigenetic perspective. J Dev Orig Health Dis (2013) 4(4):269-79. doi:10.1017/S2040174413000123

34. Johns JM, Noonan LR, Zimmerman LI, McMillen BA, Means LW, Walker CH, et al. Chronic cocaine treatment alters social/aggressive behaviour in SpragueDawley rat dams and in their prenatally exposed offspring. Ann N Y Acad Sci (1998) 846:399-404. doi:10.1111/j.1749-6632.1998.tb09765.x

35. Williams SK, Johns JM. Prenatal and gestational cocaine exposure: effects on the oxytocin system and social behaviour with implications for addiction. Pharmacol Biochem Behav (2013) 119:10-21. doi:10.1016/j.pbb.2013.07.004

36. Francis DD, Champagne FC, Meaney MJ. Variations in maternal behaviour are associated with differences in oxytocin receptor levels in the rat. J Neuroendocrinol (2000) 12(1):1145-8. doi:10.1046/j.1365-2826.2000.00599.x

37. Henriques TP, Szawka RE, Diehl LA, de Souza MA, Correa CN, Aranda BC, et al. Stress in neonatal rats with different maternal care backgrounds: monoaminergic and hormonal responses. Neurochem Res (2014) 39(12):2531-9. doi:10. 1007/s11064-014-1434-8

38. Kojima S, Stewart RA, Demas GE, Alberts JR. Maternal contact differentially modulates central and peripheral oxytocin in rat pups during a brief regime of mother-pup interaction that induces a filial huddling preference. J Neuroendocrinol (2012) 5:831-40. doi:10.1111/j.1365-2826.2012.02280.x
39. Bales KL, Perkeybile AM. Developmental experiences and the oxytocin receptor system. Horm Behav (2012) 61(3):313-9. doi:10.1016/j.yhbeh.2011.12.013

40. Clark CL, St John N, Pasca AM, Hyde SA, Hornbeak K, Abramova M, et al. Neonatal CSF oxytocin levels are associated with parent report of infant soothability and sociability. Psychoneuroendocrinology (2013) 38(7):1208-12. doi:10.1016/j.psyneuen.2012.10.017

41. Feldman R. Oxytocin and social affiliation in humans. Horm Behav (2012) 61(3):380-91. doi:10.1016/j.yhbeh.2012.01.008

42. Feldman R, Gordon I, Zagoory-Sharon O. The cross-generation transmission of oxytocin in humans. Horm Behav (2010) 58(4):669-76. doi:10.1016/j.yhbeh. 2010.06.005

43. Wismer Fries AB, Ziegler TE, Kurian JR, Jacoris S, Pollak SD. Early experience in humans is associated with changes in neuropeptides critical for regulating social behaviour. Proc Natl Acad Sci U S A (2005) 102(47):17237-40. doi:10.1073/pnas.0504767102

44. Tietz A, Zietlow AL, Reck C. Maternal bonding in mothers with postpartum anxiety disorder: the crucial role of subclinical depressive symptoms and maternal avoidance behaviour. Arch Womens Ment Health (2014) 17(5):433-42. doi:10.1007/s00737-014-0423-x

45. Forman DR, O'Hara MW, Stuart S, Gorman LL, Larsen KE, Coy KC. Effective treatment for postpartum depression is not sufficient to improve the developing mother-child relationship. Dev Psychopathol (2007) 19(2):585-602.

46. Perry DF, Ettinger AK, Mendelson T, Le HN. Prenatal depression predicts postpartum maternal attachment in low-income Latina mothers with infants. Infant Behav Dev (2011) 34(2):339-50. doi:10.1016/j.infbeh.2011.02.005

47. Glover V. Maternal depression, anxiety and stress during pregnancy and child outcome; what needs to be done. Best Pract Res Clin Obstet Gynaecol (2014) 28(1):25-35. doi:10.1016/j.bpobgyn.2013.08.017

48. Mah BL, Bakermans-Kranenburg MJ, Van Ijzendoorn MH, Smith R. Oxytocin promotes protective behaviour in depressed mothers: a pilot study with the enthusiastic stranger paradigm. Depress Anxiety (2014) 32(2):76-81. doi:10.1002/da.22245

49. Eapen V, Dadds M, Barnett B, Kohlhoff J, Khan F, Radom N, et al. Separation anxiety, attachment and interpersonal representations: disentangling the role of oxytocin in the perinatal period. PLoS One (2014) 17:9. doi:10.1371/journal. pone. 0107745

50. van der Knaap LJ, Riese H, Hudziak JJ, Verbiest MM, Verhulst FC, Oldehinkel AJ, et al. Glucocorticoid receptor gene (NR3C1) methylation following stressful events between birth and adolescence. The TRAILS study. Transl Psychiatry (2014) 4:e381. doi:10.1038/tp.2014.22

51. Champagne FA. Epigenetic mechanisms and the transgenerational effects of maternal care. Front Neuroendocrinol (2008) 29(3):386-97. doi:10.1016/j.yfrne. 2008.03.003

52. Gregory SG, Connelly JJ, Towers AJ, Johnson J, Biscocho D, Markunas CA, et al. Genomic and epigenetic evidence for oxytocin receptor deficiency in autism. BMC Med (2009) 7:62. doi:10.1186/1741-7015-7-62

53. Cecil CA, Lysenko LJ, Jaffee SR, Pingault JB, Smith RG, Relton CL, et al. Environmental risk, oxytocin receptor gene (OXTR) methylation and youth callous-unemotional traits: a 13-year longitudinal study. Mol Psychiatry (2014) 19(10):1071-7. doi:10.1038/mp.2014.95

54. Crockford C, Deschner T, Ziegler TE, Wittig RM. Endogenous peripheral oxytocin measures can give insight into the dynamics of social relationships: a review. Front Behav Neurosci (2014) 8:68. doi:10.3389/fnbeh. 2014.00068

55. Carson DS, Berquist SW, Trujillo TH, Garner JP, Hannah SL, Hyde SA, et al. Cerebrospinal fluid and plasma oxytocin concentrations are positively correlated and negatively predict anxiety in children. Mol Psychiatry (2014). doi:10.1038/mp.2014.132

56. Wittig RM, Crockford C, Deschner T, Langergraber KE, Ziegler TE, Zuberbühler K. Food sharing is linked to urinary oxytocin levels and bonding in related and unrelated wild chimpanzees. Proc Biol Sci (2014) 281:1778. doi:10.1098/rspb.2013.3096

57. Saito A, Hamada H, Kikusui T, Mogi K, Nagasawa M, Mitsui S, et al. Urinary oxytocin positively correlates with performance in facial visual search in unmarried males, without specific reaction to infant face. Front Neurosci (2014) 8:217. doi:10.3389/fnins.2014.00217

58. Szeto A, McCabe PM, Nation DA, Tabak BA, Rossetti MA, McCullough ME, et al. Evaluation of enzyme immunoassay and radioimmunoassay methods for 
the measurement of plasma oxytocin. Psychosom Med (2011) 73(5):393-400. doi:10.1097/PSY.0b013e31821df0c2

59. Neumann ID, Landgraf R. Balance of brain oxytocin and vasopressin: implications for anxiety, depression, and social behaviours. Trends Neurosci (2012) 35(11):649-59. doi:10.1016/j.tins.2012.08.004

Conflict of Interest Statement: The authors declare that the research was conducted in the absence of any commercial or financial relationships that could be construed as a potential conflict of interest.

Received: 04 November 2014; accepted: 24 February 2015; published online: 11 March 2015.
Citation: Alves E, Fielder A, Ghabriel N, Sawyer Mand Buisman-Pijlman FTA (2015) Early social environment affects the endogenous oxytocin system: a review and future directions. Front. Endocrinol. 6:32. doi: 10.3389/fendo.2015.00032

This article was submitted to Neuroendocrine Science, a section of the journal Frontiers in Endocrinology.

Copyright (c) 2015 Alves, Fielder, Ghabriel, Sawyer and Buisman-Pijlman. This is an open-access article distributed under the terms of the Creative Commons Attribution License (CC BY). The use, distribution or reproduction in other forums is permitted, provided the original author(s) or licensor are credited and that the original publication in this journal is cited, in accordance with accepted academic practice. No use, distribution or reproduction is permitted which does not comply with these terms. 